\title{
Modeling and Control for Dynamic Positioned Marine Vessels in Drifting Managed Sea Ice
}

\author{
Øivind Kåre Kjerstad Roger Skjetne
}

Department of Marine Technology, Norwegian University of Science and Technology, N-7491 Trondheim, Norway. E-mail: \{ oivind.k.kjerstad, roger.skjetne\} @ntnu.no

\begin{abstract}
This paper presents a development framework for dynamic positioning control systems for marine vessels in managed ice. Due to the complexity of the vessel-ice and ice-ice interactions a configurable high fidelity numerical model simulating the vessel, the ice floes, the water, and the boundaries is applied. The numerical model is validated using experimental data and coupled with a control application incorporating sensor models, control systems, actuator models, and other external dynamics to form a closed loop development platform. The ice drift reversal is simulated by moving the positioning reference frame in an elliptic trajectory, rather than moving each individual ice floe. A control plant model is argued, and a control system for managed ice is proposed based on conventional open water design methods. A case study shows that dynamic positioning in managed ice is feasible for some moderate ice conditions.
\end{abstract}

Keywords: Dynamic positioning, Numerical simulation, Control design, Nonlinear systems

\section{Introduction}

According to Gautier et al. (2009) the Arctic region holds approximately $30 \%$ of the undiscovered gas, and $13 \%$ of the undiscovered oil in the world. Such resources coupled with diminishing supplies of conventional oil and higher oil prices have resulted in an intensified focus on research and development work to qualify, enable, and mature technological solutions required for Arctic offshore operations. One of the major challenges is the fact that many of the undrilled prospectives lies below $100 \mathrm{~m}$ water-depth (Hamilton, 2011). To reach these requires floating prouction units that are capable of operating in the harsh Arctic environemnt. A key system enabling this is the stationkeeping system that ensures that the vessel maintains position within a given operational area. Stationkeeping ranges from passive moored structures which do not have its own actuation to dynamic positioning (DP) where a onboard control system calculates the thruster actuation needed to automatically maintain position (fixed location or predetermined track) in the presence of external disturbances (IMO, 1994). Although floating production units in the Arctic are foreseen to be thruster assisted moored structures, where a control system works together with the moorings to enable efficient and robust positioning, DP will be an essential component during the exploration phase, connection and disconnection from the mooring, and for support and intervention.

When vessels interacts with high concentrations of sea ice (above 6/10th), the environmental forces are substantially different and conventional open-water DP systems are known to be insufficient (Kerkeni et al., 2013; Gürtner et al., 2012; Hals and Jenssen, 2012; Jenssen et al., 2009). Nevertheless, it has been demonstrated by full-scale, model-scale, and numerical experiments that high-uptime positioning is possible with ice management (IM) support (Rohlén, 2009; Keinonen and Martin, 2012; Hals and Jenssen, 2012; Metrikin et al., 2013; Liferov, 2014). IM involves all aspects of removing or reducing ice actions on the protected 


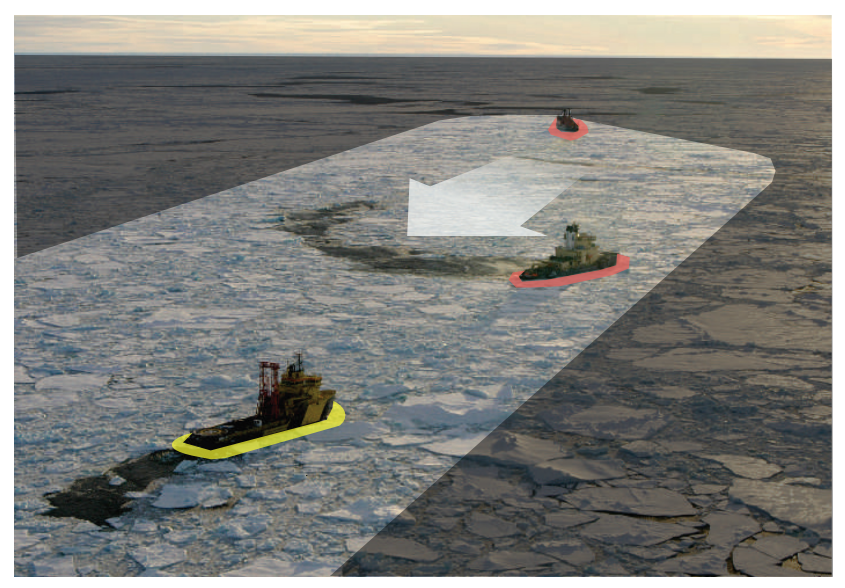

Figure 1: Ice management concept featuring the protected DP vessel (enveloped in yellow) and the physical ice management fleet (enveloped in red). Adapted from (Moran et al., 2006).

vessel (Eik, 2010). Actual IM activities are operationspecific, but the main objective is to either transform the natural ice environment into an acceptable managed ice condition or suspend the operation if that is not possible. Figure 1 illustrates a modern physical IM operation: the operational fleet, the ice cover, and the ice drift direction.

During development of control systems, models of the vessel dynamics are needed for simulation and control systems design. Sørensen (2012) defines two levels of model fidelity: process plant models and control plant models. The process plant model describes the actual physics involved with high fidelity and replaces costly deployments during development and testing. There are several benefits to computer simulations, but most notably it enables rapid design iterations in a controllable and repeatable environment. The control plant model is typically a simplification of the process plant model capturing the important characteristics of the process. It is the foundation of model-based control design and used in analytical stability analysis. This is usually obtained by identifying the plant dynamics from first principles and experimental data.

Today, the development of ice-enabled DP systems heavily depend on experimental testing and tuning in laboratory basins (see for instance (Hals and Efraimsson, 2011) and (Jenssen et al., 2012)) where a model scale vessel controlled by a DP system is propelled through an ice cover frozen and cut to match the ice conditions. Figure 2 shows a DP test at the Hamburg Ship Model Basin (HSVA). Although model ice tanks create realistic ice environments for linear motion, testing of phenomena or maneuvers requiring some transversal space is challenging. Also, the over-

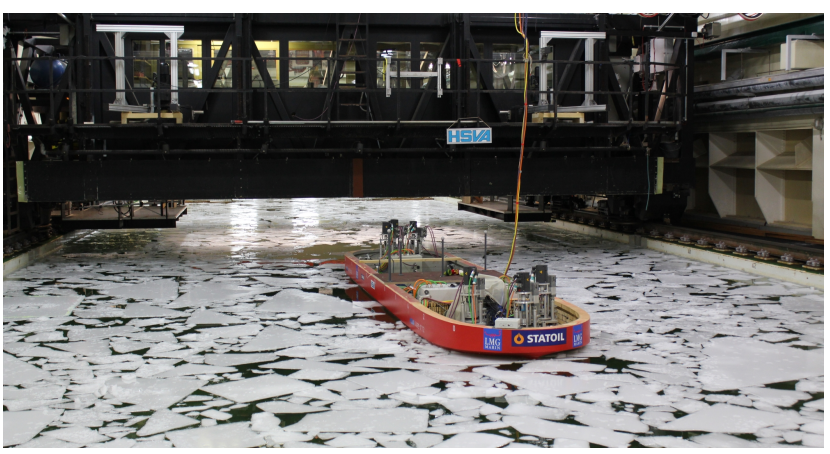

Figure 2: A vessel at DP in the large ice basin at HSVA during DYPIC. Courtesy of DYPIC.

head and expenses involved with each experiment is high, and each frozen ice cover is constrained to a few tests with limited repeatability. Therefore, applying simulation models relieving laboratory experiments is considered highly beneficial.

This paper presents a development platform for DP in ice which applies the numerical model presented in (Metrikin, 2014) and data from the European research project DYnamic Positioning In iCe (DYPIC). The latter was a 3 year initiative (2010-2012) led by HSVA and financed by the national research agencies of Germany, France, and Norway. The program focused on various aspects of DP technology for offshore operations in ice-infested waters, where almost 250 different scenarios were performed in broken ice conditions in the large ice tank of the HSVA (which is $72 \mathrm{~m}$ long and $10 \mathrm{~m}$ wide). Comprehensive project overviews can be found in (Jenssen et al., 2012) and (Kerkeni et al., 2014).

The contribution of this paper is: (1) description, application, and investigation of a numerical tool as a process model for control system development in managed ice; (2) simulation of ice drift reversals by elliptic trajectories; (3) derivation of a control design model and control algorithms for DP in managed sea-ice; and (4) investigation of the proposed design by a case study.

\subsection{Scope}

The planar 3 degrees of freedom (DOF) equations of motion of a DP vessel are:

$$
\begin{aligned}
\dot{\boldsymbol{\eta}} & =\mathbf{R}(\psi) \boldsymbol{\nu} \\
\mathbf{M}_{R B} \dot{\boldsymbol{\nu}} & =\boldsymbol{\tau}+\boldsymbol{\tau}_{\text {hydro }}+\boldsymbol{\tau}_{\text {wind }}+\boldsymbol{\tau}_{\text {waves }}+\boldsymbol{\tau}_{\text {ice }}
\end{aligned}
$$

where $\boldsymbol{\eta} \in \mathbb{R}^{3}$ is the position and orientation vector expressed in an inertial frame, $\mathbf{R}(\psi) \in \mathbb{R}^{3 \times 3}$ is the rotation matrix between the inertial frame and the body frame where $\psi \in \mathbb{R}$ is the vessel heading, $\boldsymbol{\nu} \in \mathbb{R}^{3}$ is the body frame velocity vector, $\mathbf{M}_{R B} \in \mathbb{R}^{3 \times 3}$ is the 


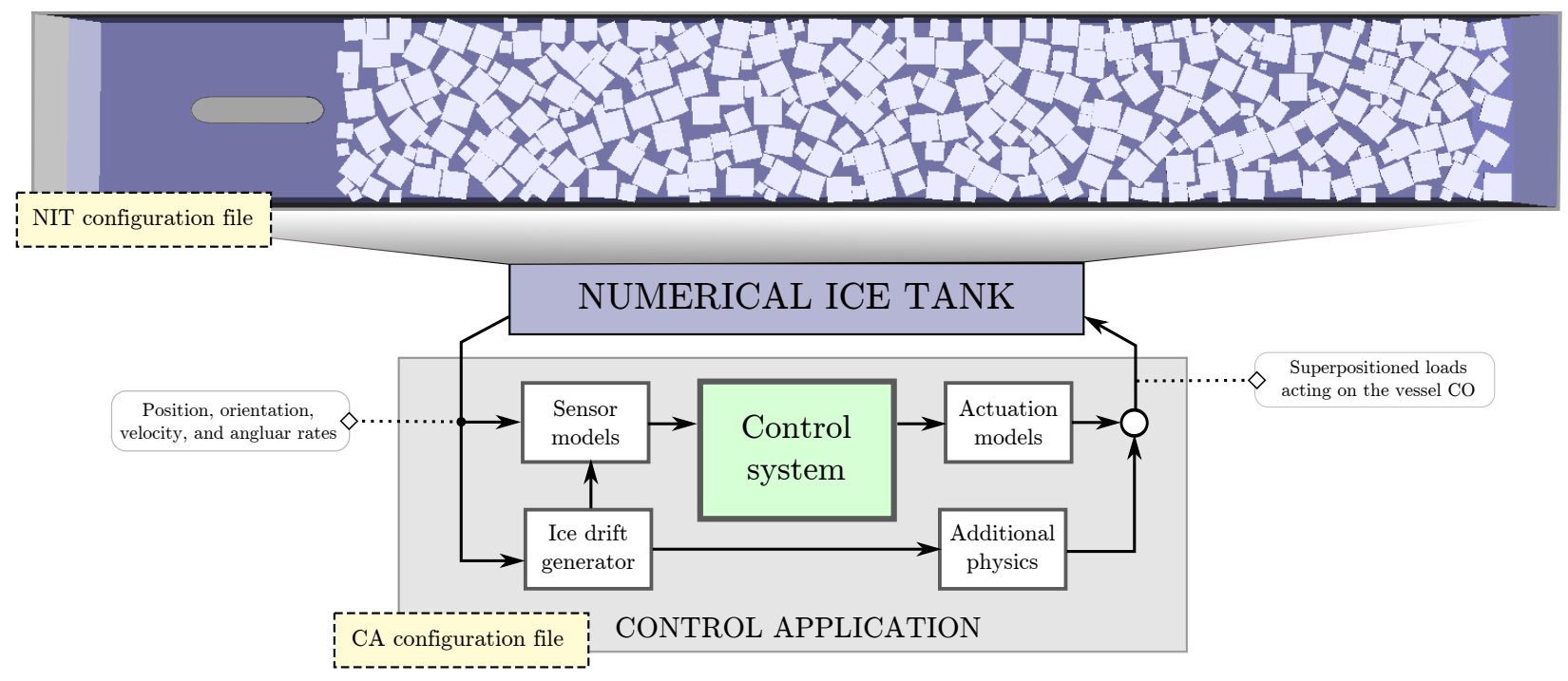

Figure 3: The modular structure of the closed-loop simulation platform for DP in managed ice.

rigid body mass matrix, $\boldsymbol{\tau} \in \mathbb{R}^{3}$ is the vessel actuation, $\boldsymbol{\tau}_{\text {hydro }} \in \mathbb{R}^{3}$ is inertial, hydrodynamic, and hydrostatic loads (including current loads), $\boldsymbol{\tau}_{\text {wind }} \in \mathbb{R}^{3}$ is the wind loads, $\boldsymbol{\tau}_{\text {waves }} \in \mathbb{R}^{3}$ is the wave loads, and $\boldsymbol{\tau}_{\text {ice }} \in \mathbb{R}^{3}$ is the ice loads from the interaction with the managed ice cover. In this paper the variable notation found in (SNAME, 1950) is applied.

Unfortunately, no analytical dynamic models for the managed ice loads $\boldsymbol{\tau}_{i c e}$ exist (Eik, 2010), and deriving one able to relate the in-situ load variations to measurable variables is challenging (Kjerstad et al., 2014). This mainly affects the derivation of a control design model for managed ice. Numerical methods have shown promise as process models when simulating each individual ice floe as a body (see for instance (Metrikin et al., 2013) and (Metrikin, 2014)). Therefore, the first problems treated in this paper are:

1. Apply and validate a numerical managed ice model as a process model and simulation framework for DP in managed ice;

2. Establish a control design model for DP control systems development in managed ice.

Although DP in managed ice have been proven feasible, the control algorithms have not been exposed. Also, it is uncertain which elements of the operation is most challenging for the DP control system. Thus, the final problem treated here is:

3. Adapt conventional DP control systems technology for operations in managed ice and illustrate challenges involved with the operation.

\section{Process Model and Closed Loop Simulation Platform}

Numerical models based on first principles describing the vessel and the ice floes as interacting bodies with their respective spatial geometries and material properties are considered necessary to simulate the complex vessel-ice and ice-ice interactions and capture the time varying load transients. As managed ice can consist of high amounts of coupled ice floes, the computational complexity of simulating these may also become high. However, models applying physics engines have shown great promise for handling this, see e.g. (Lubbad and Løset, 2011),(Metrikin et al., 2012b,a, 2013).

As mentioned above, the numerical model described in (Metrikin, 2014) is applied as the process model for DP in managed ice. It is implemented as a fixed timestep model based on nonsmooth 6 degrees of freedom (DOF) multi-body dynamics with contacts, friction, and material properties. In the following it will be referred to as the Numerical Ice Tank (NIT), and Section 2.2 elaborates further on its capabilities and validity. Each simulation is comprised of the following five interconnected elements: the rigid body vessel, the towing carriage, the ice floes, the water volume, and the tank boundaries. The NIT can be set up in three different simulation modes:

- Free running, where the vessel is forced through the ice cover by a fixed input force and moment vector.

- Towing, where the vessel is forced through the ice cover by the carriage at a given velocity. 
- $D P$, where the vessel is propelled by a body force and moment vector defined at each time step.

For development of DP control systems the latter two modes are used. Towing is applied to study the ice dynamics without the additional vessel dynamics and calibrate the numerical model through replicating HSVA ice basin experiments. The DP mode is used to close the loop between the simulator vessel motion output and the body force and moment vector to test various control algorithms. Figure 3 provides an overview the closed-loop simulation platform.

\subsection{Kinematics}

The NIT vessel motion variables are defined in two reference frames:

- The tank-fixed frame $\{t\}$ which is non-rotational and fixed to the stationary tank boundaries;

- The body frame $\{b\}$ is fixed to the vessel body.

For development of DP a third reference frame is introduced to simulate ice drift in the numerical ice tank:

- The positioning frame $\{n\}$ which is non-rotational and following a pre-defined trajectory to simulate ice drift in the stationary ice cover. The DP vessel will be set to track a fixed position in this frame.

Figure 4 illustrates the reference frames. In this paper $\{n\}$ is considered inertial. The main reason for this approximation is that it is common in ice tank testing (Haase and Jochmann, 2013), and thereby enables a basis for comparison for calibration and validation. The position and orientation vector $\boldsymbol{\eta}$ given in the $\{n\}$ frame relates to the $\{\mathrm{t}\}$ frame as

$$
\boldsymbol{\eta}=\boldsymbol{\eta}_{t}-\boldsymbol{\eta}_{i d}
$$

where $\boldsymbol{\eta}_{t} \in \mathbb{R}^{3}$ is the position and orientation vector in the tank frame, and $\boldsymbol{\eta}_{i d} \in \mathbb{R}^{3}$ is the position of $\{\mathrm{n}\}$ in $\{\mathrm{t}\}$. The dynamics of $\boldsymbol{\eta}_{i d}$ will be treated in-depth in Section 2.4. The body frame $\{b\}$ relates to $\{n\}$ as

$$
\dot{\boldsymbol{\eta}}=\mathbf{R}(\psi) \boldsymbol{\nu}
$$

\subsection{The Numerical Ice Tank}

As the NIT is treated in-depth in (Metrikin, 2014), only a brief summary of its capabilities relevant for describing the closed-loop simulation platform is given.

The NIT computes the vessel dynamics in 6 DOF without wind and waves, using a physics engine with tailored routines for handling the ice material properties. Each simulation is comprised of the following five

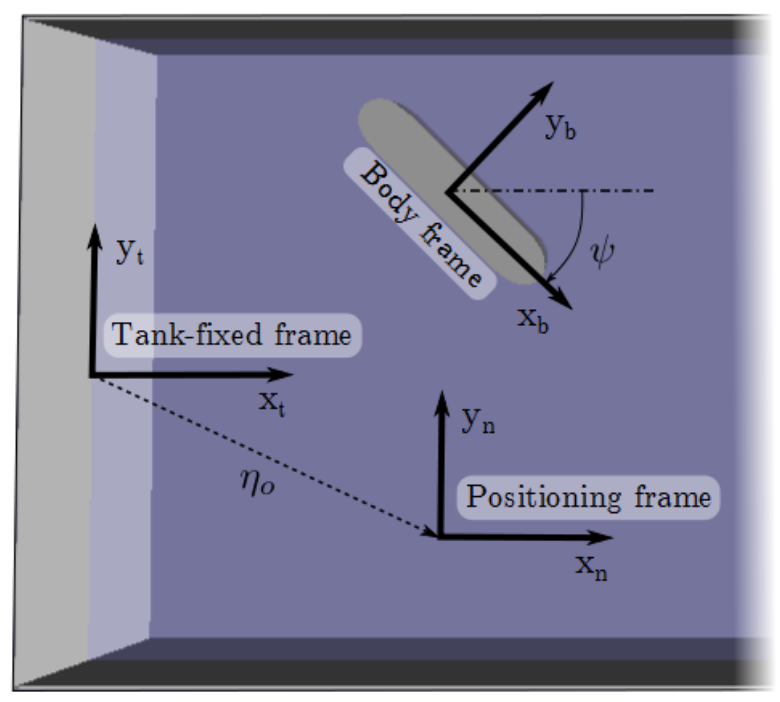

Figure 4: The reference frames of the closed loop simulation platform for DP in managed ice.

interconnected elements: the vessel, the towing carriage, the ice floes, the water volume, and the tank boundaries. The vessel is simulated as a rigid body in 6 DOF without deformations. Throughout this paper a 3D model of an Arctic Drillship (ADS), which was experimentally tested in DYPIC, is used (see Section 4.1). The towing carriage is simulated by a prismatic joint that restricts the motions of the vessel in $5 \mathrm{DOF}$ and allows only the heave displacement. As the towing progresses, the prismatic joint measures the global loads on the vessel from the ice and the fluid by calculating the constraint forces in all DOFs. This mimics the six-component scale commonly used in model testing. It should be noted that the towing carriage is only included in the simulation in towing mode.

The ice floes are simulated as breakable bodies with uniform thickness in 6 DOF. The initial ice floe sizes and floe positions are generated by a ice field generation algorithm that aims to produce a specificed ice field. The water is simulated as a static plane that produces buoyancy and drag loads on the vessel and the ice floes. Tables 1 and 2 summarize the processes in the NIT. The tank boundaries are simulated as stationary rigid bodies. The interface to the model is a XML file that specifies vessel model properties, tank dimensions, ice field properties, ice material properties, water properties, and boundary properties.

\subsection{Calibration and Precision}

To calibrate the NIT and evaluate its precision the DYPIC towing experiments shown in Table 3 were 

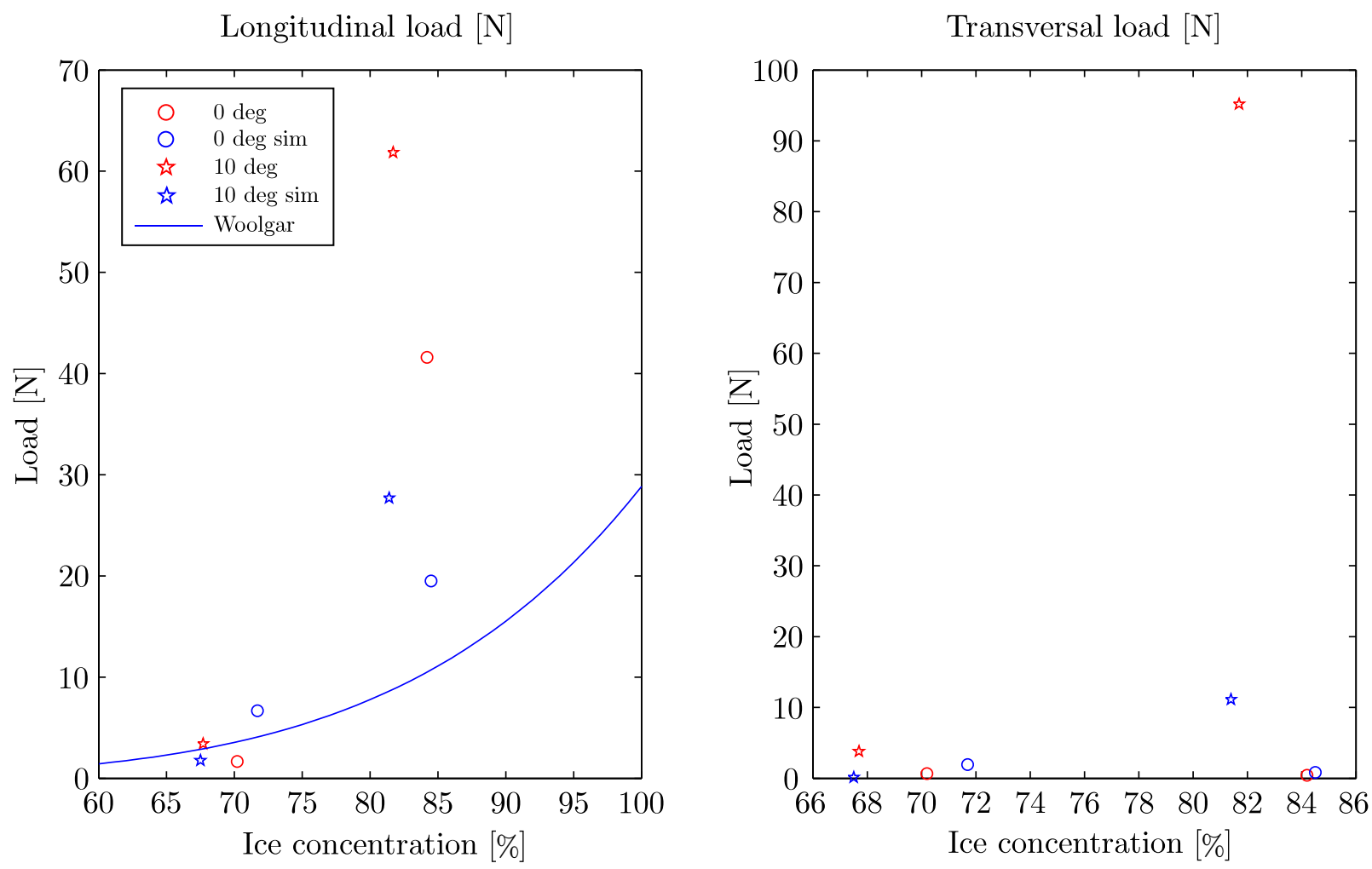

Figure 5: Comparison between model scale data of the experiments seen in Table 3, numerical simulations, and the empirical formula found in (Woolgar and Colbourne, 2010).

Table 1: NIT physical processes and coefficients.

\begin{tabular}{lc}
\hline Physical process & Coef. value \\
\hline \hline Ice-ice static friction & 0.2 \\
Ice-ice dynamic friction & 0.2 \\
Ice-wall static friction & 0.2 \\
Ice-wall dynamic friction & 0.2 \\
Ice-vessel static friction & 0.0976 \\
Ice-vessel dynamic friction & 0.0976 \\
Linear form drag coefficient & 0.4 \\
Angular form drag coefficient & 0.6 \\
Skin friction & 0.01 \\
\hline
\end{tabular}

replicated. Towing experiments simplify the comparison and interpretation of the simulation results with the experimental data as it is independent of vessel dynamics (and control system). In this study the dynamic friction coefficients of Table 1 were identified from experiments in the ice laboratory at HSVA. In reality this normally differs from the static friction coefficients. However, the NIT applies the same value for both dynamic and static friction. The linear and angular form drag coefficients, and the skin friction were found by trial and error. As it is not possible to exactly replicate the experimental ice floe configuration of the
Table 2: Simulator parameters.

\begin{tabular}{lcc}
\hline Parameters & Symbol & Unit \\
\hline \hline Water density & $\rho_{w}$ & $\mathrm{~kg} / \mathrm{m}^{3}$ \\
Ice density & $\rho_{c}$ & $\mathrm{~kg} / \mathrm{m}^{3}$ \\
Ice flexural strength & $\sigma_{f}$ & $\mathrm{kPa}$ \\
Ice compressive strength & $\sigma_{c}$ & $\mathrm{kPa}$ \\
Ice elastic modulus & $E$ & $\mathrm{Mpa}$ \\
Ice concentration & $c$ & $\%$ \\
Ice thickness & $h$ & $\mathrm{~mm}$ \\
\hline
\end{tabular}

ice cover in the NIT (floe positions and orientations), an ice cover with the same properties was used. See Figure 6 for a comparison. Further, the elastic modulus was set to $10 \mathrm{MPa}$ for all runs, the water and ice densities were set to 1000 and $900 \mathrm{~kg} / \mathrm{m}^{3}$, respectively.

Figure 5 shows the correspondence between experimental data, numerical simulations, and the empirical formula for pack ice loads on stationkeeping vessels for the presented parameters. This shows that the longitudinal load is replicated better in the NIT than using the empirical formula. However, the match of the NIT from the experimental data is seemingly deviating as the ice concentration and oblique angle increases. This is especially evident in the transversal loads. There 
Table 3: DYPIC towing experiments. Exp. denotes the experiment number, $\psi_{r}$ in $[d e g]$ is the oblique angle, $v_{r}$ in $[\mathrm{m} / \mathrm{s}]$ is the vessel velocity in $\{\mathrm{t}\}, c$ is the ice concentration, $h$ is the ice thickness, $\sigma_{f}$ is the ice flexural strength, and $\sigma_{c}$ is the ice compressive strength.

\begin{tabular}{ccccccc}
\hline Exp. & $\psi_{r}$ & $v_{r}$ & $c$ & $h$ & $\sigma_{f}$ & $\sigma_{c}$ \\
\hline \hline 4100 & 170 & $0.023,0.047$ & 81.7 & 28.7 & 60.8 & 92 \\
4200 & 170 & $0.023,0.047$ & 67.7 & 28.7 & 45.9 & 92 \\
5100 & 180 & $0.023,0.047$ & 84.2 & 24.3 & 64.3 & 89 \\
5200 & 180 & $0.023,0.047$ & 70.2 & 24.3 & 56.8 & 89 \\
\hline
\end{tabular}

may be a number of interconnected reasons for this, but pinpointing the actual ones are challenging because the NIT is an integrated environment where many physical processes are coupled. Thus, care must be taken in both experimental design and results interpretation. However, it must be mentioned that the precision can be improved by tuning the linear and angular form drag coefficients for each individual experiment.

The main advantage of the NIT is that it captures two of the fundamental vessel-ice and ice-ice processes described in (Kjerstad et al., 2014): Ice floe contact networks and accumulation of ice mass. Both relates to the behavior of the drifting ice cover when an obstructing vessel is present and are considered highly important to capture the in-situ time-varying dynamical behavior of the vessel and the ice. This is not captured in any other available model (i.e., statistical or empirical models).

\subsection{Closed-Loop Simulation Platform}

The control application (CA) of Figure 3 is a collection of interconnected models which enable testing of control algorithms during execution of the NIT (in DP mode). Although it's specific implementation depends on the control system in question, it is divided into the following five general modules: sensor models, ice drift generator, control system, actuator models, and additional physics.

The sensor models simulate onboard equipment measuring the vessel motion, consisting of a coordinate transformation and sensor modeling. First, the NIT vessel motion output (position, orientation, linear velocity, and angular rate) is transformed from $\{\mathrm{t}\}$ to $\{\mathrm{n}\}$ and $\{\mathrm{b}\}$, and then sensor dynamics and noise is added to the signals. Besides the NIT motion variables, signals originating from the other $\mathrm{CA}$ components are possible. The specific sensor implementations are dependent on the application and sensor characteristics.

The ice drift generator models the ice drift in the basin. Typically, linear ice drift, where the vessel tracks

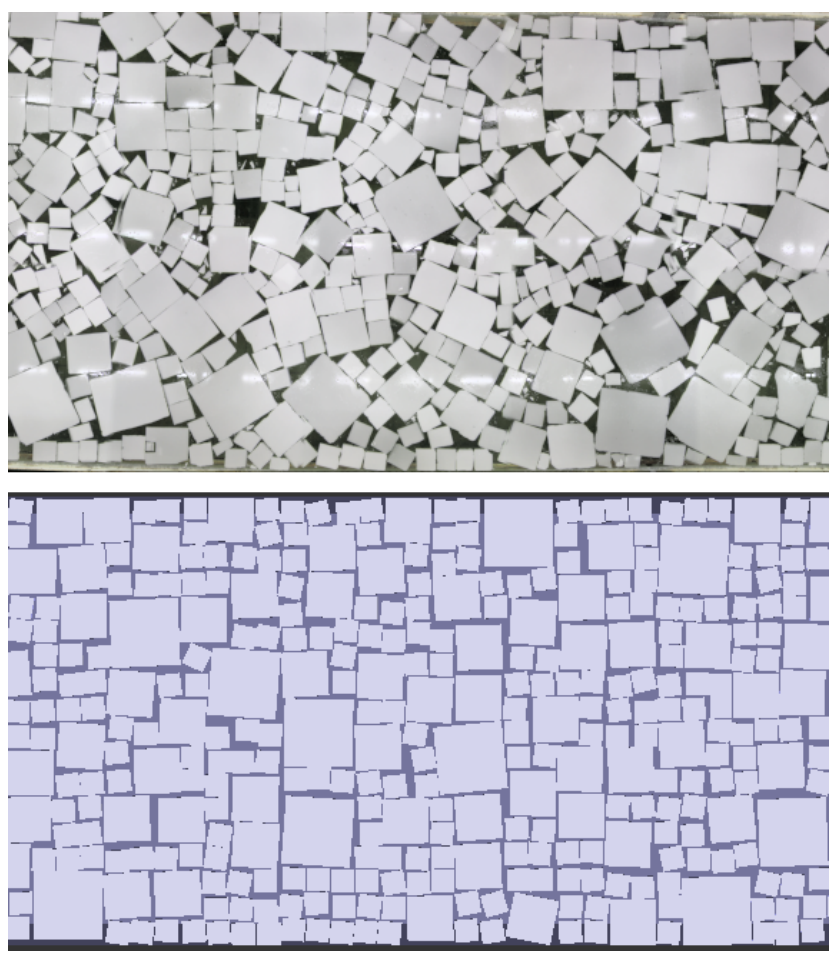

Figure 6: A comparison between the HSVA ice cover and the NIT ice cover for experiment 4100.

a point moving from one side of the basin to the other is considered. However, Keinonen and Martin (2012) reports that the ice drift reversals originating from tides and Coriolis forcing are a major challenge to positioning. Therefore, such a drift scenario is considered in this paper. From the drift patterns in Figure 8 an elliptic trajectory was selected. This is realized by the following motion of $\{n\}$ in $\{t\}$,

$$
\boldsymbol{\eta}_{i d}=\boldsymbol{\eta}_{0}+\boldsymbol{\eta}_{e}
$$

where $\boldsymbol{\eta}_{0} \in \mathbb{R}^{3}$ is the initial position of $\{\mathrm{n}\}$ and $\boldsymbol{\eta}_{e} \in \mathbb{R}^{3}$ describes the elliptic trajectory as

$$
\begin{aligned}
\dot{\boldsymbol{\eta}}_{e} & =\boldsymbol{\Psi} \\
\dot{\boldsymbol{\Psi}} & =\left[\begin{array}{c}
-a \alpha^{2} \sin (\alpha t) \\
-b \alpha^{2} \cos (\alpha t) \\
0
\end{array}\right]
\end{aligned}
$$

where $a$ and $b$ are coefficients determining the spatial size of the ellipse, and $\alpha=\frac{v_{i}}{a}$ where $v_{i}$ is the maximum ice drift velocity. Figure 7 illustrates the concept. Note that only one half of the ellipse is applied, where the velocity along this decreases until the positioning frame reaches the pivot. Then, the velocity starts to increase again. This complies with observations in nature.

The control system module contains the implemen- 


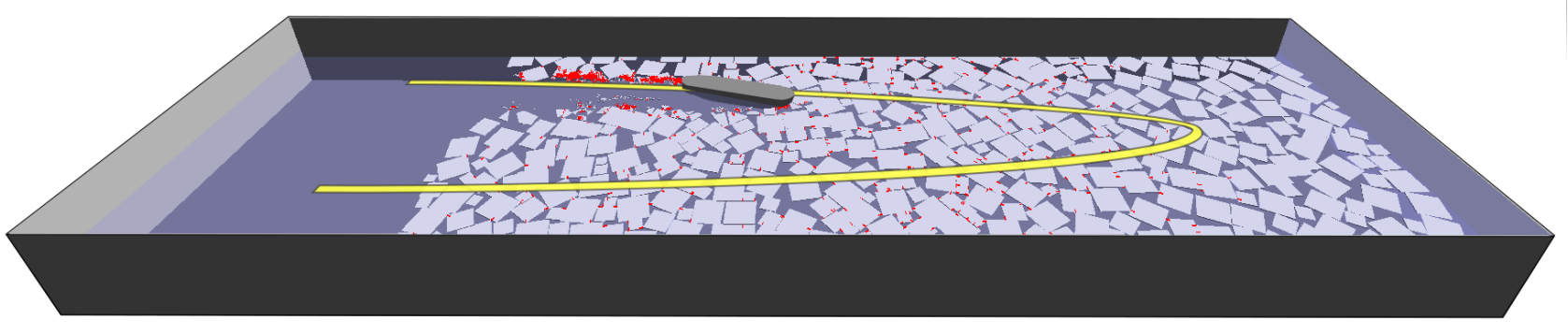

Figure 7: Illustration of the elliptic ice drift trajectory in the closed loop simulation platform.

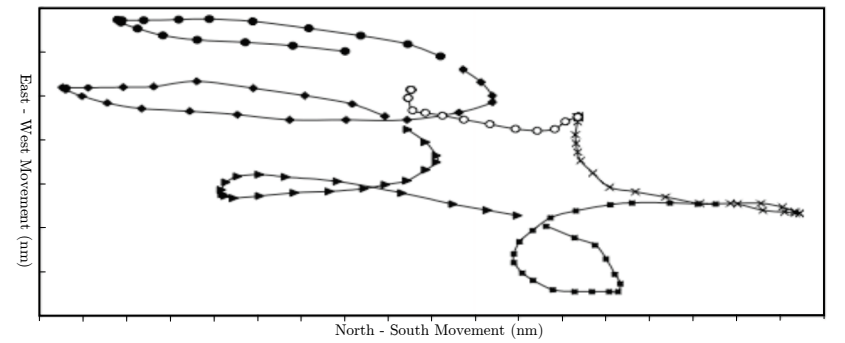

Figure 8: The reversing ice drift as reported by Keinonen et al. (2000).

tation of the control algorithms, and a specific implementation is given in Section 3. The actuator models implement the dynamics of the thruster system onboard the vessel. Experience obtained from full-scale experiments indicates that first-order models are well suited (Sørensen et al., 1996). Thus,

$$
\dot{\tau}=-\mathbf{A}_{t}^{-1}(\boldsymbol{\tau}-\mathbf{T u})
$$

where $\mathbf{A}_{t}$ is a diagonal matrix of time constants, $\mathbf{T} \in$ $\mathbb{R}^{3 \times 12}$ is the thruster configuration matrix, and $\mathbf{u}$ is the commanded thrust input from each individual thruster.

The additional physics module contains vessel specific dynamics which are not modeled explicitly in the NIT. Examples of such are wind loads, additional vessel drag, and mooring loads. These are added to the body force and moment vector together with the actuation forces. However, neither was implemented in this study.

It is important to note that the closed-loop system does not enable models implemented in the CA to apply forces on other bodies in the simulation than the vessel. For instance, the thruster wake which is known to affect the ice cover, is not captured. Depending on the vessel and it thruster system, this may be of importance. Therefore, care must be taken when interpreting the simulation results.

\subsubsection{Implementation and Experimental Design Considerations}

The NIT comes as an object file library (.lib). For convenience, the $\mathrm{CA}$ is also generated as an object file library from it's initial implementation in Matlab/Simulink. Both the NIT and the CA libraries are accessed by a $\mathrm{C}++$ interface. Using this, a simulation executable is created. The simulator pseudo code is seen in Algorithm 1.

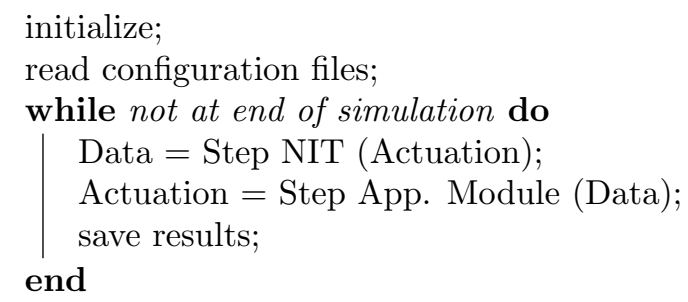

Algorithm 1: The closed-loop simulator's main program loop.

Although the closed-loop simulator can generate a wide range of scenarios, there are two experimental design considerations, besides the ones already mentioned, that must be recognized:

1. The number of bodies in the simulation. This will affect the run-time of the simulation as packed ice field will be computationally heavier than lower concentration scenarios.

2. The boundary conditions. These can severely impact the load dynamics if the ice floe contact networks of the vessel interacts with them. However, in some cases this may be a part of the experimental design, i.e., stationkeeping in a narrow managed ice channel.

Judging the capabilities of the closed-loop simulation platform it is found to be a feasible for early development and testing of conceptual control algorithms for specific and confined maneuvers. With respect to load 

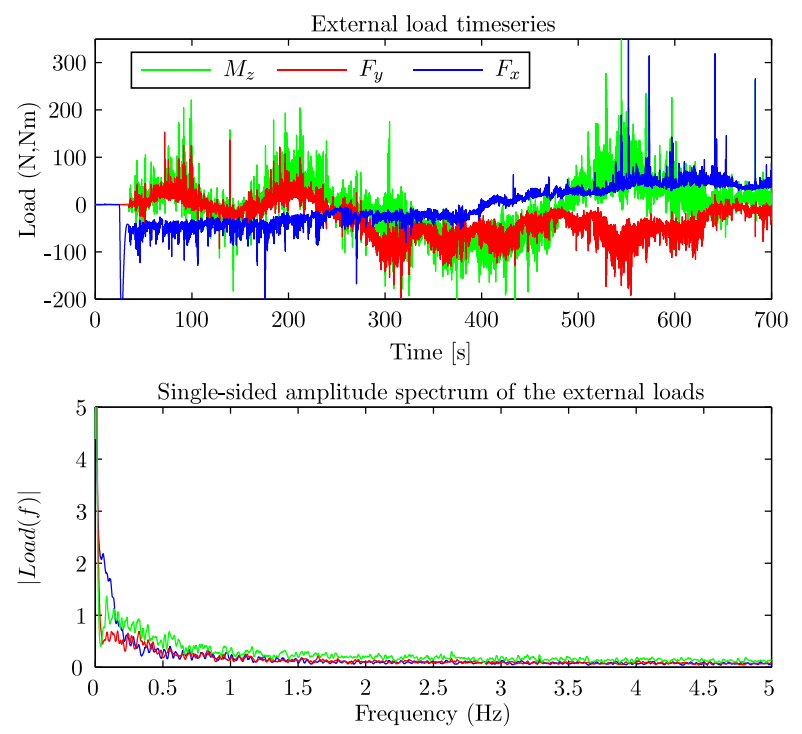

Figure 9: The time-varying nature and frequency spectrum of the external loads of the NIT. The spectrum is low-pass filtered using a moving average filter to show trends.

precision and validity, the applicability depends on the objective of the experiment. For instance, if the precision of the loads is critical, such as for tuning, then the range of applicable ice conditions are lower than for proof-of-concept simulations where the objective may be investigating stability and parameter sensitivity.

\section{DP Control System Design}

In this section a control design model is derived and used to synthesize a DP control system for managed ice. Both the model and control system consider the planar motion of the vessel; surge, sway, and yaw. Further, it is assumed that the velocity range of DP is sufficiently low such that phenomena such as centripetal forces and Coriolis effects can be neglected.

\subsection{Ice Loads and the Control Plant Model}

To design a model-based control law a control design model description of (1)-(2) is needed. For open water this is established and several model exist (see (Fossen, 2011)). In this study the following model is chosen as a starting point,

$$
\begin{aligned}
\dot{\boldsymbol{\xi}}= & \mathbf{A}_{w} \boldsymbol{\xi}+\mathbf{E}_{w} \mathbf{w}_{w} \\
\dot{\boldsymbol{\eta}}= & \mathbf{R}(\psi) \boldsymbol{\nu} \\
\dot{\mathbf{b}}_{o}= & \mathbf{w}_{o} \\
\mathbf{M} \dot{\boldsymbol{\nu}}= & \boldsymbol{\tau}-\mathbf{D} \boldsymbol{\nu}+\mathbf{R}(\psi)^{\top} \mathbf{b}_{o} \\
& +\boldsymbol{\tau}_{\text {wind }}+\boldsymbol{\tau}_{i c e}+\mathbf{w}_{v}
\end{aligned}
$$

where $\boldsymbol{\xi} \in \mathbb{R}^{6}$ is a first order wave response state, $\mathbf{A}_{w} \in \mathbb{R}^{6 \times 6}$ and $\mathbf{E}_{w} \in \mathbb{R}^{6 \times 3}$, are constant matrices describing the sea state, and $\mathbf{M}:=\mathbf{M}_{R B}+\mathbf{M}_{a}$ where $\mathbf{M}_{a}$ is the added mass from accelerating the fluid around the ships hull. Notice that the rotation matrix in (10) only depends on the heading $\psi$. This comes fo the assumption that roll and pitch angles of the vessel are small. The bias term $\mathbf{b}_{o} \in \mathbb{R}^{3}$ is a non-physical quantity that incorporates several effects such as ocean current loads, modeling errors, unmodeled dynamics, and wave drift. The variables $\mathbf{w}_{i}(i=w, o, v)$ are zero-mean Gaussian noise vectors representing model uncertainty and measurement noise. $\boldsymbol{\tau} \in \mathbb{R}^{3}$ is the control input, and $\boldsymbol{\tau}_{\text {wind }} \in \mathbb{R}^{3}$ is the wind loads (assumed measured). See Fossen (2011) for further information.

Figure 9 shows the external loads (hydrodynamics and ice) from one closed-loop simulation. The frequency analysis shows that the loads appear in the low end of the spectrum. This complies well with the findings in (Kjerstad et al., 2014), and imply that integral control may be sufficient to deal with the disturbances.

Although it has been found that the mean ice loads on a stationkeeping vessel depend on the oblique angle, ice concentration, ice properties, floe size distribution, and ice thickness, no applicable dynamic model describing the time variations exists (Eik, 2010; Kjerstad et al., 2014). There are several reasons for this. Mainly, the signals have high variance and rapid transients (as seen in Figure 9) that are not described well by neither the given ice parameters nor the vessel states. This stems from the fact that the in-situ configuration, dynamics, and boundary conditions of the ice floe contact networks heavily impact the load. Nevertheless, the load may be divided into the following components,

$$
\boldsymbol{\tau}_{i c e}=\boldsymbol{\tau}_{i}+\boldsymbol{\tau}_{f}+\boldsymbol{\tau}_{p},
$$

where $\boldsymbol{\tau}_{i}$ are inertial load components that originate from the added ice mass which the vessel must actuate and the de-acceleration of incoming ice floes, $\boldsymbol{\tau}_{f}$ is additional friction originating from hydrodynamic phenomena on the ice floes, vessel-ice friction, and ice-ice friction. $\boldsymbol{\tau}_{p}$ is loads from large scale ice cover pressure.

Although (13) is rooted in highly complex and multibody nature it seems reasonable to assume that the ice induces both added inertia and friction to the dynamic system. Thus, a mass-damper model can be argued

$$
\begin{aligned}
& \boldsymbol{\tau}_{i c e}=-\mathbf{M}_{i c e} \dot{\boldsymbol{\nu}}+\mathbf{d}_{i c e}(\boldsymbol{\nu})+\mathbf{R}^{\top} \mathbf{b}_{i c e}+\mathbf{w}_{i c e} \\
& \dot{\mathbf{b}}_{i c e}=\mathbf{w}_{b},
\end{aligned}
$$

where $\mathbf{M}_{i c e} \in \mathbb{R}_{>0}^{3 \times 3}$ is added ice mass and $\mathbf{d}_{\text {ice }}(\boldsymbol{\nu}) \in \mathbb{R}^{3}$ is a damping function describing the ice condition, $\mathbf{b}_{i c e}$ is a Wiener process, and $\mathbf{w}_{b}$ and $\mathbf{w}_{i c e}$ are zero- 
mean Gaussian noise vectors. A major downside of this model is the fact that $\mathbf{M}_{i c e}$ and $\mathbf{d}_{i c e}(\boldsymbol{\nu})$ are highly dependent on the in-situ ice floe contact networks, accumulated ice mass, and boundary conditions which requires them to be determined on-line if to be used in control. This adds complexity to system in the form of additional integrators. However, if a high quality ice load measurement is available this approach may be feasible, as seen in (Østhus, 2014). If no such signal is available, and alternative is to linearize $\mathbf{d}_{i c e}(\boldsymbol{\nu}) \approx \mathbf{D}_{i c e} \boldsymbol{\nu}$ and use fixed $\mathbf{M}_{i c e}$ and $\mathbf{D}_{i c e}$ matrices. However, practice has shown that estimating these matrices off-line is challenging and no significant improvement in tracking capabilities is gained with respect to incorporating all ice loads in one bias estimate such as,

$$
\begin{aligned}
\boldsymbol{\tau}_{i c e} & =\mathbf{R}(\psi)^{\top} \mathbf{b}_{i c e}+\mathbf{w}_{i c e} \\
\dot{\mathbf{b}}_{i c e} & =\mathbf{w}_{b} .
\end{aligned}
$$

Therefore, (16)-(17) is selected as the design model in the remainder of this study.

Another aspect of the ice environment is the exponential attenuation of high frequency oscillatory wave loads (Broström and Christensen, 2008). This allows for removing the wave model (9). For the subsequent control design this will be beneficial as it reduces the number states and model uncertainty. Hence, the following adaptation of (9)-(12) can be applied as a control plant model for managed ice,

$$
\begin{aligned}
\dot{\boldsymbol{\eta}} & =\mathbf{R}(\psi) \boldsymbol{\nu} \\
\dot{\mathbf{b}} & =\mathbf{w}_{1} \\
\mathbf{M} \dot{\boldsymbol{\nu}} & =-\mathbf{D} \boldsymbol{\nu}+\boldsymbol{\tau}+\boldsymbol{\tau}_{\text {wind }}+\mathbf{R}(\psi)^{\top} \mathbf{b}+\mathbf{w}_{2}
\end{aligned}
$$

where $\mathbf{b}=\mathbf{b}_{o}+\mathbf{b}_{i c e}$. For simplicity this model can be written in compact for as

$$
\dot{\mathbf{x}}=\mathbf{A}(\psi) \mathbf{x}+\mathbf{B} \boldsymbol{\zeta}+\mathbf{E w}
$$

where $\mathbf{x}=\left[\begin{array}{lll}\boldsymbol{\eta} & \boldsymbol{\nu} & \mathbf{b}\end{array}\right]^{\top} \in \mathbb{R}^{9}$ is the state vector and $\boldsymbol{\zeta}=\left[\begin{array}{ll}\boldsymbol{\tau} & \boldsymbol{\tau}_{\text {wind }}\end{array}\right]^{\top} \in \mathbb{R}^{3 \times 2}$ is a input vector. $\mathbf{A}(\psi) \in$ $\mathbb{R}^{9 \times 9}, \mathbf{B} \in \mathbb{R}^{3 \times 2}$, and $\mathbf{E} \in \mathbb{R}^{9 \times 3}$ are matrices describing the system according to (18)-(20).

\subsection{Control Design}

For the control design we apply the conventional DP approach found in for instance (Fossen, 2011) and (Sørensen, 2012), where the main goal is to develop a control law that can be used to investigate and pinpoint elements that need further development. To achieve this, two sensor suites are considered:

1. Position and heading measurements only.
2. Position, heading, linear velocity, and angular rate measurements.

In conventional DP control systems the linear velocity measurement is not commonly considered. These signals are here assumed available through technologies such as GNSS Doppler systems and inertially aided hydroacustic positioning systems. However, it is still considered necessary to employ a controller-observer structure where the observer will provide sensor fusion, filter measurements, estimate the bias state, and provide dead-reckoning in the case of measurement loss. Thus, we consider the following components:

- Observer

- Control law and reference system

- Control allocation

\subsubsection{Observer}

Traditionally, either a Kalman filter or a nonlinear passive DP observer (Fossen, 2011) is used. As the tuning parameters of the latter are more intuitively connected to the physics of the system (Sørensen, 2012) this approach is selected. Copying (18)-(20) and introducing an injection term gives,

$$
\dot{\hat{\mathbf{x}}}=\mathbf{A}(\psi) \hat{\mathbf{x}}+\mathbf{B} \boldsymbol{\xi}+\mathbf{L}(\psi)(\mathbf{y}-\mathbf{C} \hat{\mathbf{x}}),
$$

where $\mathbf{L}(\psi) \in \mathbb{R}^{9 \times n}$ is a nonlinear injection gain matrix, and $n$ is either 3 or 6 depending on the sensor suite. For the second sensor suite, $\mathbf{L}(\psi)$ is proposed as

$$
\mathbf{L}(\psi)=\left[\begin{array}{cc}
\mathbf{K}_{1} & \mathbf{K}_{4} \mathbf{R}(\psi) \\
\mathbf{K}_{2} \mathbf{R}(\psi)^{\top} & \mathbf{K}_{5} \\
\mathbf{K}_{3} & \mathbf{K}_{6} \mathbf{R}(\psi)
\end{array}\right] .
$$

UGAS stability of the proposed observer is established using the Lyapunov function candidate $V=\mathbf{x}^{\top} \mathbf{P} \mathbf{x}$, where $\mathbf{P}=\mathbf{P}^{\top}>0$. For the first sensor suite, $\mathbf{L}(\psi)$ becomes the first column of (23), and becomes identical to the nonlinear passive DP observer. UGAS is ensured by Theorem 11.2 in (Fossen, 2011).

Both Kerkeni et al. (2013) and Jenssen et al. (2009) report deficient performance of open water observers in managed ice experiments. Judging the proposed observers it is clear that the only option to improve the tracking performance is to apply more aggressive tuning than what is common in open water. Especially for the bias estimate. However, this approach is constrained by the the quality of the measurements. If high injection gains are used with noisy measurements the estimation performance may deteriorate. 


\subsubsection{Control Law and Reference Model}

The control law determines the 3 DOF generalized forces and moment that is required for fulfilling the control objectives (tracking a fixed location or a predefined path). Traditionally, the structure of the control law is a nonlinear PID with anti-windup of the integral action (Sørensen, 2012). In this study the windup routines are disregarded for simplicity, and the control structure is chosen as

$$
\boldsymbol{\tau}=\boldsymbol{\tau}_{F F}+\boldsymbol{\tau}_{F B},
$$

where $\boldsymbol{\tau}_{F F}$ are feedforward terms and $\boldsymbol{\tau}_{F B}$ are feedback terms. These are assigned as

$$
\begin{aligned}
\boldsymbol{\tau}_{F F}= & \mathbf{M} \dot{\boldsymbol{\nu}}_{d}-\boldsymbol{\tau}_{\text {wind }} \\
\boldsymbol{\tau}_{F B}= & -\mathbf{K}_{p} \mathbf{R}(\psi)^{\top} \tilde{\boldsymbol{\eta}}-\mathbf{K}_{d} \tilde{\boldsymbol{\nu}} \\
& -\mathbf{K}_{i} \mathbf{R}(\psi)^{\top} \int_{0}^{t} \tilde{\boldsymbol{\eta}} d t
\end{aligned}
$$

where $\tilde{\boldsymbol{\eta}}:=\hat{\boldsymbol{\eta}}-\boldsymbol{\eta}_{d}$ is the position error, $\tilde{\boldsymbol{\nu}}:=\hat{\boldsymbol{\nu}}-\boldsymbol{\nu}_{d}$ is the velocity error, and $\mathbf{M} \dot{\boldsymbol{\nu}}_{d}$ constitutes a desired motion feedforward. $\boldsymbol{\eta}_{d}, \boldsymbol{\nu}_{d}$, and $\dot{\boldsymbol{\nu}}_{d}$ are determined using a third order reference filter for the setpoint.

\subsubsection{Control Allocation}

DP vessels are usually over-actuated which means that the vessel has more actuators than degrees of freedom. This requires a control allocation algorithm to determine the output each actuator such that the generalized control vector $\boldsymbol{\tau}$ is produced. The following linear mapping is applied

$$
\tau=\mathbf{T u}
$$

where $\mathbf{u} \in \mathbb{R}^{n}$ is a vector of thrust forces and $\mathbf{T} \in \mathbb{R}^{3 \times n}$ it the thruster configuration matrix, where $n$ is the number of thrust components. Here, the allocation algorithm found in (Skjetne and Kjerstad, 2013) is used.

\section{Simulation Case Study}

In this case study, DP subject to managed ice drift reversals in medium and high ice concentration is investigated. This is done to shed light on the proposed control system's performance and understand the relation to the severity of the ice condition.

\subsection{The Arctic Drillship}

The vessel applied is the conceptual ADS seen in Figure 2. This is optimized for operations in ice and experimentally tested in the DYPIC project. The vessel has three azimuth thrusters in the bow and three in the stern, making it suitable for DP. Its main particulars and specific azimuth thruster arrangement are found in tables 4 and 5. Further description of the ADS is found in (Gürtner et al., 2012; Hals and Jenssen, 2012; Metrikin et al., 2013; Kjerstad et al., 2014).

Table 4: ADS main particulars and illustration of the thruster arrangement.

\begin{tabular}{lc}
\hline Parameter & Model scale \\
\hline \hline Length in design waterline $(\mathrm{m})$ & 6.67 \\
Length between perpendiculars $(\mathrm{m})$ & 6.13 \\
Breath, modeled $(\mathrm{m})$ & 1.37 \\
Draught at design waterline $(\mathrm{m})$ & 0.4 \\
Stem angle at design waterline $\left(^{\circ}\right)$ & 45 \\
Frame angle at midship $\left(^{\circ}\right)$ & 45 \\
Displacement volume $\left(\mathrm{m}^{3}\right)$ & 2535 \\
Center of gravity from aft. perp. $(\mathrm{m})$ & 3.18 \\
Block coefficient & 0.75 \\
Metacentric height $(\mathrm{m})$ & 0.357 \\
Total thrust $(\mathrm{N})$ & 201 \\
\hline
\end{tabular}

Table 5: The ADS azimuth thruster arrangement.

\begin{tabular}{clccc}
\hline No. & Comment & $\mathrm{x}[\mathrm{mm}]$ & $\mathrm{y}[\mathrm{mm}]$ & $\mathrm{F}[\mathrm{N}]$ \\
\hline \hline 1 & Port-Bow & 2272 & 316 & 22 \\
2 & Center-Bow & 2644 & 0 & 22 \\
3 & Stb-Bow & 2272 & -316 & 22 \\
4 & Center-Stern & -3102 & 0 & 45 \\
5 & Port-Stern & -2664 & 190 & 45 \\
6 & Stb-Stern & -2664 & -190 & 45 \\
\hline
\end{tabular}

The proposed control design of Section 3 require the matrices $\mathbf{M}$ and $\mathbf{D}$ to be determined. Here, the rigid body matrix is applied as the system inertia matrix $\mathbf{M}$. This follows directly from the vessel's dry mass and is

$$
\mathbf{M}=\operatorname{diag}\left(\left[\begin{array}{lll}
2535 & 2535 & 8485
\end{array}\right]\right) .
$$

Commonly in DP control design, the applied system inertia matrix is a summation of the dry mass and an approximation of the added mass gained from accelerating fluid around the hull. However, since the added mass effects are not incorporated in the NIT it is not considered in this study.

D were approximated experimentally in the NIT by running the vessel to steady state in open water using constant input. This resulted in

$$
\mathbf{D}=\operatorname{diag}\left(\left[\begin{array}{lll}
75 & 100 & 3205
\end{array}\right]\right) .
$$

\subsection{Experiment Setup}

The two cases considered were: 
Table 6: State estimator and control law gains.

\begin{tabular}{cccc}
\hline Gain & Surge & Sway & Yaw \\
\hline \hline$K_{1}$ & 4 & 4 & 4 \\
$K_{2}$ & 1000 & 1500 & 5000 \\
$K_{3}$ & 30 & 60 & 500 \\
$K_{4}$ & 1 & 1 & 1 \\
$K_{5}$ & 1000 & 1500 & 5000 \\
$K_{6}$ & 400 & 750 & 4000 \\
$K_{p}$ & 200 & 200 & 400 \\
$K_{d}$ & 1000 & 1000 & 3000 \\
$K_{i}$ & 6 & 6 & 14 \\
\hline
\end{tabular}

- Case 1: Elliptic drift reversal in 55\% managed ice.

- Case 2: Elliptic drift reversal in $80 \%$ managed ice.

In both cases the ice strength properties and floe size distribution were set to replicate experiment 4100 (see Table 3$)$ in a $75 \times 18 \times 2.5 \mathrm{~m}$ basin. The ice drift ellipse parameters in (7) were set to $a=45$ and $b=4.5$ with a maximum ice drift of $0.2 \mathrm{~m} / \mathrm{s}$. Gaussian white noise with standard deviations of $0.01 \mathrm{~m}$ (and $\mathrm{m} / \mathrm{s}$ ) were used for the position and linear velocity measurements. The standard deviation for the heading and angular rate measurements were $0.1 \mathrm{deg}$ (and $\mathrm{deg} / \mathrm{s})$. The control system gains seen in Table 6 were obtained by trial and error in order to achieve a feasible trade-off between noise filtering, state tracking, setpoint tracking, and thruster usage. The closed-loop bandwidth was found to be $f_{b}=\left[\begin{array}{lll}1.83 & 1.83 & 1.45\end{array}\right] \mathrm{Hz}$. With respect to Figure 9 this is well above the main frequency range of the external loads.

Three simulations were performed based in the two sensor suites described above: Case 1 with sensor suite 1 , case 2 with sensor suite 1 , and case 2 with sensor suite 2. All were performed with identical initial conditions, where the control objective is to track $\{\mathrm{n}\}$ keeping the heading constant at $\psi=0$. Effectively this means tracking a fixed location. The positioning frame was initialized at $\boldsymbol{\eta}_{0}=\left[\begin{array}{lll}10.5 & -4.5 & 0\end{array}\right]^{\top}$ in $\{\mathrm{t}\}$. Wind loads were not included in the simulations. To quantify the performance of the observers, the following cost function is applied

$$
Q(t)=\int_{0}^{t} \tilde{\mathbf{x}}^{\top} \mathbf{W} \tilde{\mathbf{x}}
$$

where $\tilde{\mathbf{x}} \in \mathbb{R}^{9}$ is the observer error state, and $\mathbf{W} \in$ $\mathbb{R}^{9 \times 9}$ is a static diagonal positive definite normalization matrix. (30) gives the cumulative error of the state estimates as a function of time, where low value indicates high precision.

\subsection{Results and Discussion}

Figure 10 shows the simulation results where it can be seen that the positioning and heading keeping capabilities in $55 \%$ ice concentration is fair. One important reason for this is that the vessel deflects and pushes incoming ice floes away and no severe ice floe contact networks nor ice mass accumulation forms. Thus, the load variations are contained to minor perturbations which are well tracked and counteracted. In $80 \%$ ice concentration these phenomena are more evident causing a severe load regime. This is indirectly shown by the control input norms for case 2, and causes performance deterioration of the suite 1 control system. The main reason for this is the fact that the point of attack and magnitude of the ice loads are constantly changing. This creates a continous change of the vessel momentum which must be sensed though the double time integrals of the force (position and heading measurements). As the momentum is already gained when it materialized in the measurements it causes a sustained transient deviation in the observer (for all states). In turn it causes inaccurate thruster output calculation and results in poor positioning. Therefore, it makes sense to incorporate velocity and angular rate measures as these capture the momentum earlier. This is verified by the case 2 : suite 2 results. With the NIT capabilities in mind, it may be that the ice loads are underpredicted causing a somewhat optimistic positioning performance for both runs of case 2. Nevertheless, the ice concentration dependency complies well with reports of open-water DP systems, which work in light conditions, but struggles as the ice condition toughens (see (Keinonen et al., 2006) and (Rohlén, 2009)). It should also be noted that the conditions toughens as the oblique angle increases. This is seen in the tank frame position plot of Figure 10.

The heading tracking of the vessel does not show the same degree of improvement as the positioning in case 2: suite 2. A combination of sub-optimal control law (both in its structure and tuning) and the brute force operation strategy, resulting in sustained high oblique angle for most of the simulation, is believed to be the main reasons for this. In general, if allowed by the operation, the DP vessel should act to prevent the oblique angle from becoming high.

In sum this study indicates that classical DP control architecture with the proposed modifications is feasible for conditions without high ice concentration. It is also believed to extend the operational window with respect to conventional open-water systems. This is achieved mainly through removing the wave filter from the observer, re-tuning it and the control law more aggressively, and when available adopting velocity and angular rate measurements. Still, it is uncertain whether 

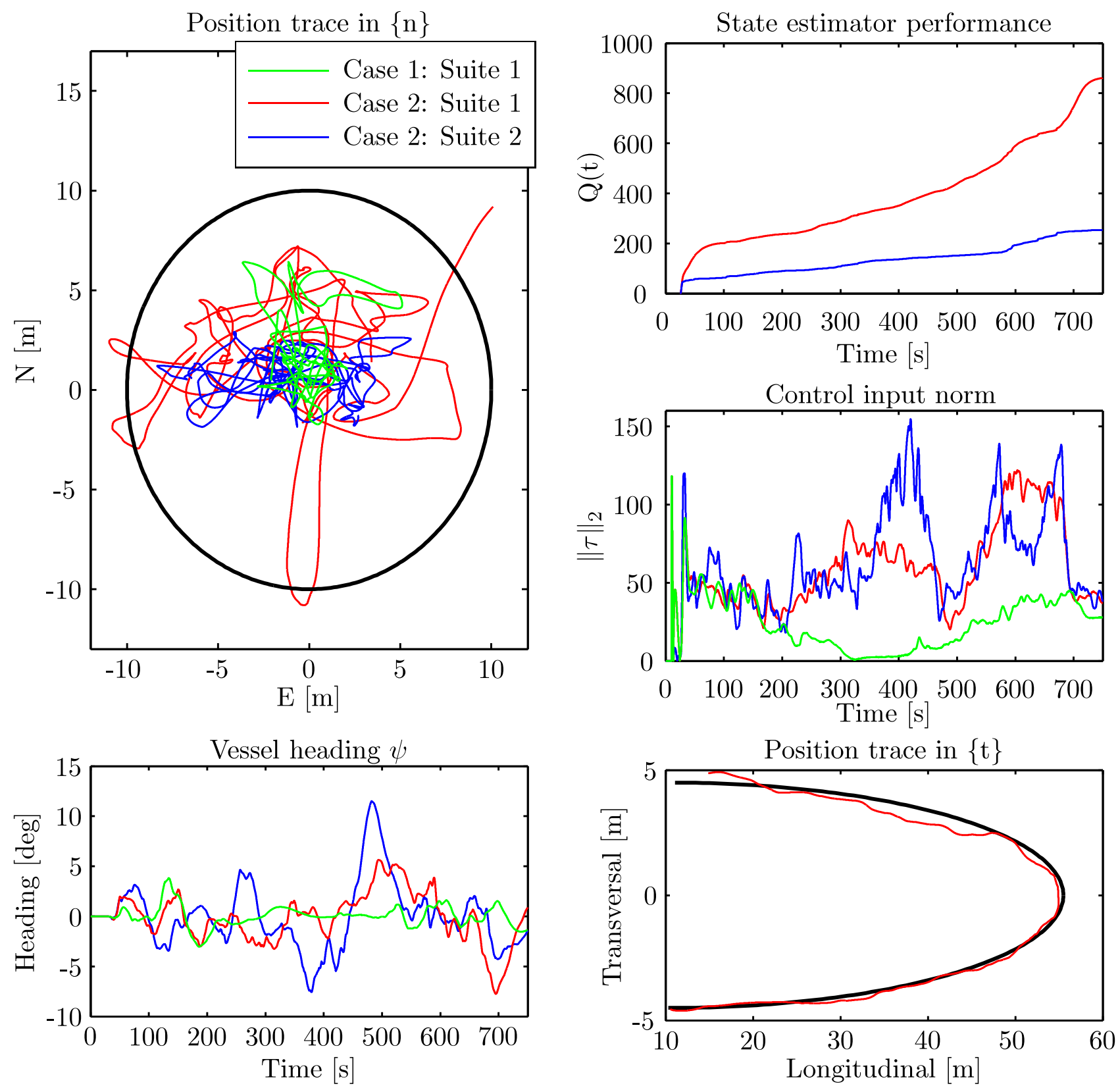

Figure 10: A comparison of the two control systems setups. The positioning frame trace plot is scaled to full scale using $\lambda=30$. The observer performance plot shows the performance measure for case 2 : suite 1 , where the suite 2 observer was run in parallel. The initial position of the vessel in $\{\mathrm{t}\}$ was $x=10.5$ $\mathrm{m}$ and $y=-4.5 \mathrm{~m}$. 
this provides sufficient reactivity to handle high ice concentrations, ice pressure, and large or abnormal ice features slipping through the IM. Thus, it may be required to improve the reactivity of the system further and implement proactive ice handling measures to take on such conditions. Kjerstad et al. (2014) provides an overview of some possible improvements.

\section{Summary and Conclusions}

This paper have described and motivated the use of a given numerical tool as a process model for developing DP control systems. Its precision exceeds that of a comparable empirical model, but some underprediction is seemingly occurring at high ice concentrations and oblique angles. The strength of the model, in DP development context, is its ability to capture the time-varying variation in the loads from processes in the ice cover. This is considered key for testing control systems.

A development framework closing the loop of the numerical model was presented. This incorporates important components such as sensors characteristics, actuator dynamics, and ice drift. For the latter, an elliptic trajectory was chosen to model both the temporal and spatial dynamics.

Ice-adapted control algorithms were proposed based on a control design model. The ice dynamics were included in the observer bias estimate, and handled using a conventional nonlinear PID control law as no ice specific parameter nor vessel state were considered to describe the load variations. In summary, what separates the proposed enhancements from conventional open-water systems is the removal of the wave filter, and aggressive system tuning. The control system was tested using the developed simulation platform where it showed an ice concentration dependency on the positioning capability. Incorporating additional vessel velocity and angular rate measurements aided this to some extent.

\section{Acknowledgments}

Research partly funded by the Research Council of Norway (RCN) KMB project no. 199567: "Arctic DP", with partners Kongsberg Maritime, Statoil, and DNV GL, and partly by RCN project no. 203471: CRI SAMCoT. Additionally, the authors would like to thank Nils Albert Jenssen and Lars Imsland for constructive feedback, and Ivan Metrikin for providing the numerical model, technical support, and constructive feedback.

\section{References}

Broström, G. and Christensen, K. Waves in sea ice. Technical report, Norwegian Meteorological Institute, Norway, 2008.

Eik, K. J. Ice Management in Arctic Offshore Operations and Field Developments. Ph.D. thesis, NTNU, Norway, 2010.

Fossen, T. I. Handbook of Marine Craft Hydrodynamics and Motion Control. Wiley, 2011. doi:10.1002/9781119994138.

Gautier, D. L., Bird, K. J., Charpentier, R. R., Grantz, A., Houseknecht, D. W., Klett, T. R., Moore, T. E., Pitman, J. K., Schenk, C. J., Schuenemeyer, J. H., Sørensen, K., Tennyson, M. E., Valin, Z. C., and Wandrey, C. J. Assessment of Undiscovered Oil and Gas in the Arctic. Science, 2009. 324(5931):11751179. doi:10.1126/science.1169467.

Gürtner, A., Baardson, B. H. H., Kaasa, G.-O., and Lundin, E. Aspects of importance related to Arctic DP operations. In Proc. 31th Int. Conf. on Ocean, Offshore and Arctic Engineering (OMAE). 2012. doi:10.1115/OMAE2012-84226.

Haase, A. and Jochmann, P. Different ways of modeling ice drift scenarios in basin tests. In Proc. 32th Int. Conf. on Ocean, Offshore and Arctic Engineering (OMAE). 2013. doi:10.1115/OMAE2013-10793.

Hals, T. and Efraimsson, F. DP Ice Model Test of Arctic Drillship. In Proc. of the Dynamic Positioning Conference. 2011.

Hals, T. and Jenssen, N. A. DP ice model tests of Arctic drillship and polar research vessel. In Proc. 31th Int. Conf. on Ocean, Offshore and Arctic Engineering. 2012. doi:10.1115/OMAE2012-83352.

Hamilton, J. M. The Challenges of Deep-Water Arctic Development. Int. Journal of Offshore and Polar Engineering, 2011. 21(4):241-247.

International Maritime Organization (IMO). Guidelines for Vessels with Dynamic Positioning Systems. 1994. MSC/circ.645.

Jenssen, N. A., Hals, T., Haase, A., Santo, X., Kerkeni, S., Doucy, O., Gürtner, A., Hetschel, S. S., Moslet, P. O., Metrikin, I., and Løset, S. A Multi-National R\&D Project on DP Technology in Ice. In Proc. of the Dynamic Positioning Conference. 2012.

Jenssen, N. A., Muddesitti, S., Phillips, D., and Backstrom, K. DP In Ice Conditions. In Proc. of the Dynamic Positioning Conference. 2009. 
Keinonen, A. and Martin, E. H. Modern day pioneering and its safety in the floating ice offshore. In Proc. 10th Int. Conf. and Exhibition on Performance of Ships and Structures in Ice (ICETECH), volume 1. 2012.

Keinonen, A., Shirley, M., Liljeström, G., and Pilkington, R. Transit and Stationary Coring Operations in the Central Polar Pack. In Proc. 7th Int. Conf. and Exhibition on Performance of Ships and Structures in Ice (ICETECH). 2006.

Keinonen, A., Wells, H., Dunderdale, P., Pilkington, R., Miller, G., and Brovin, A. Dynamic positioning operation in ice, offshore Sakhalin, May-June 1999. In Proc. of the 10th Int. Offshore and Polar Engineering Conf. (ISOPE). 2000.

Kerkeni, S., Dal Santo, X., and Metrikin, I. Dynamic Positioning in Ice - Comparison of Control Laws in Open Water and Ice. In Proc. 32th Int. Conf. on Ocean, Offshore and Arctic Engineering (OMAE). 2013. doi:10.1115/OMAE2013-10918.

Kerkeni, S., dal Santo, X., Doucy, O., Jochmann, P., Haase, A., Metrikin, I., Løset, S., Jenssen, N. A., Hals, T., Gürtner, A., Moslet, P. O., and Støle Hetschel, S. DYPIC project: Technological and scientific progress opening new perspectives. In Proc. of the Arctic Technology Conference (ATC). 2014.

Kjerstad, Ø., Metrikin, I., Løset, S., and Skjetne, R. Experimental and phenomenological investigation of dynamic positioning in managed ice. Cold Regions Science and Technology, 2014.

Liferov, P. Station-keeping in ice - normative requirements and informative solutions. In Proc. Arctic Technology Conference (ATC). 2014.

Lubbad, R. and Løset, S. A numerical model for realtime simulation of ship-ice interaction. Cold Regions Science and Technology, 2011. 65(2):111 - 127.

Metrikin, I. A Software Framework for Simulating Stationkeeping of a Vessel in Discontinuous Ice. Modeling, Identification and Control, 2014. 35(4):211-246. doi:10.4173/mic.2014.4.2.

Metrikin, I., Borzov, A., Lubbad, R., and Løset, S. Numerical Simulation of a Floater in a BrokenIce Field - Part II: Comparative Study of Physics Engines. In Proc. 31th Int. Conf. on Ocean,
Offshore and Arctic Engineering (OMAE). 2012a. doi:10.1115/OMAE2012-83430.

Metrikin, I., Løset, S., Jenssen, N. A., and Kerkeni, S. Numerical Simulation of Dynamic Positioning in Ice. Marine Technology Society Journal, 2013. 47(2):1430. doi:10.4031/MTSJ.47.2.2.

Metrikin, I., Lu, W., Løset, S., and Kashafutdinov, M. Numerical Simulation of a Floater in a Broken-Ice Field - Part I: Model Description. In Proc. 31th Int. Conf. on Ocean, Offshore and Arctic Engineering (OMAE). 2012b. doi:10.1115/OMAE2012-83938.

Moran, K., Backman, J., and Farrell, J. W. Deepwater drilling in the Arctic Ocean's permanent sea ice. In Proc. of the Integrated Ocean Drilling Program (IODP). 2006.

Østhus, V. Robust Adaptive Control of a Surface Vessel in Managed Ice Using Hybrid Position and Force Control. Master's thesis, NTNU, Norway, 2014.

Rohlén, Å. Relationship Between Ice-Management and Station Keeping in Ice. Presentation at Dynamics Positioning Conference, 2009.

Skjetne, R. and Kjerstad, Ø. Recursive nullspacebased control allocation with strict prioritization for marine craft. In Proc. of 9th IFAC Conf. on Control Applications in Marine Systems. 2013. doi:10.3182/20130918-4-JP-3022.00052.

Sørensen, A. J. Marine Control Systems: Propulsion and Motion Control of Ships and Ocean Structures. Department of Marine Technology, NTNU, Norway, 2012 .

Sørensen, A. J., Sagatun, S. I., and Fossen, T. I. Design of a dynamic positioning system using model-based control. Control Engineering Practice, 1996. 4(3):359 - 368. doi:10.1016/0967-0661(96)00013-5.

The Society of Naval Architects Marine Engineers. Nomenclature for treating the motion of a submerged body through a fluid. Technical report, 1950. Technical and Research Bulletin, 1-5.

Woolgar, R. C. and Colbourne, D. B. Effects of hull-ice friction coefficient on predictions of pack ice forces for moored offshore vessels. Ocean Engineering, 2010. 37:296 - 303. doi:10.1016/j.oceaneng.2009.10.003. 